\title{
Parastomal hernia - current knowledge and treatment
}

\author{
Roman Styliński, Adam Alzubedi, Sławomir Rudzki \\ Department of General and Transplant Surgery and Nutritional Treatment, Medical University of Lublin, Lublin, Poland \\ Videosurgery Miniinv 2018; 13 (1): 1-8 \\ DOI: https://doi.org/10.5114/wiitm.2018.72685
}

\begin{abstract}
Intestinal stoma creation is one of the most common surgical procedures. The most common long-term complication following stoma creation is parastomal hernia, which according to some authors is practically unavoidable. Statistical differences of its occurrence are mainly due to patient observation time and evaluation criteria. Consequently, primary prevention methods such as placement of prosthetic mesh and newly developed minimally invasive methods of stoma creation are used. It seems that in the light of evidence-based medicine, the best way to treat parastomal hernia is the one that the surgeon undertaking therapy is the most experienced in and is suited to the individuality of each patient, his condition and comorbidities. As a general rule, reinforcing the abdominal wall with a prosthetic mesh is the treatment of choice, with a low rate of complications and relapses over a long period of time. The current trend is to use lightweight, large pore meshes.
\end{abstract}

Key words: hernia, colorectal surgery, parastomal hernia.

\section{Introduction}

Parastomal hernia is the most common complication in various types of stomas. It can progress almost asymptomatically, often resulting only in an abdominal deformity in the vicinity of the stoma, but in extreme cases it can lead to bowel incarceration and strangulation, thus necessitating immediate surgery. It is estimated that at present, approximately 1 in 3 patients suffering from this complication are treated surgically, which is partly explained by the high recurrence rate observed after repair [1]. This led to the emergence of the concept of primary prevention using prosthetic mesh, particularly since many experts claim that the incidence of parastomal hernia is practically unavoidable and to a considerable extent simply a matter of time [2].

\section{Definitions}

Parastomal hernia is a type of incisional hernia occurring in abdominal integuments in the vicini- ty of a stoma, i.e. a condition wherein abdominal contents, typically the bowel or greater omentum, protrude through abdominal integuments surrounded by the hernia sac at the location of formed stoma [3]. Significant problems result from the lack of a standard definition, even though as early as in 1973, Devlin suggested a classification based on 4 types of hernias [4]: type I - integumentary (socalled true parastomal hernia); type II - subcutaneous; type III - intra-stomal; and type IV - pseudo pre-stomal.

As follows from the above, not every deformity in the stomal region, especially when relatively small, is classified as a "real" parastomal hernia, but the classification itself is of little value in clinical practice [5]. As a consequence, a number of alternative classifications have been proposed: by Moreno-Matias in 2009 [6] and Seo in 2011 [7], in both cases based on computed tomography imaging, and by Szczepkowski in 2011 [8], based on physical examination of the patient (Table I).

\section{Address for correspondence}

Adam Alzubedi, Department of General and Transplant Surgery and Nutritional Treatment, Medical University of Lublin, 8 Jaczewskiego St, 20-954 Lublin, Poland, phone: +48 516155 596, e-mail: adam.alzubedi@gmail.com 
Table I. Parastomal hernia classifications

\begin{tabular}{|c|c|}
\hline Moreno-Matias (2009) and Seo (2011) & Szczepkowski (2011) \\
\hline $\begin{array}{l}0 \text { - CT image normal, peritoneum follows the wall of the } \\
\text { bowel forming the stoma, with no formation of a sac } \\
\text { la - bowel forming the colostomy with a sac of under } 5 \mathrm{~cm} \\
\text { Ib - bowel forming the colostomy with a sac of over } 5 \mathrm{~cm} \\
\text { II - sac containing omentum } \\
\text { III - sac containing an intestinal loop other than the bowel } \\
\text { forming the stoma }\end{array}$ & $\begin{array}{l}\text { I- isolated, small parastomal hernia } \\
\text { II - small parastomal hernia with coexisting midline incisional } \\
\text { hernia without any significant front abdominal wall } \\
\text { deformity } \\
\text { III - isolated, large parastomal hernia with front abdominal } \\
\text { wall deformity } \\
\text { IV - large parastomal hernia with coexisting midline incisional } \\
\text { hernia, with front abdominal wall deformity }\end{array}$ \\
\hline
\end{tabular}

Of the classifications described above, only Szczepkowski's approach took into consideration the coexistence of incisional hernia and the size of the parastomal hernia itself. Since it relied on a physical examination of the patient and included suggestions of suitable surgical treatment for the respective types of hernia, it was the most clinically usable classification. Moreover, complementing the same with diagnostic imaging would facilitate more accurate identification of the pathology and preparation of the surgery plan $[9,10]$.

Because none of the above classifications have been used in any clinical trial or cohort group description, in 2014 the European Hernia Society published a new classification based on Szczepkowski's classification to improve the ability to compare different studies and their results, which could result in developing new evidence-based therapeutic guidelines. Subclasses of classification were defined as follows: type I - smaller than $5 \mathrm{~cm}$ in diameter without coexisting incisional hernia; type II - smaller than $5 \mathrm{~cm}$ in diameter with coexisting incisional hernia; type III - larger than $5 \mathrm{~cm}$ in diameter without coexisting incisional hernia; and type IV - larger than $5 \mathrm{~cm}$ in diameter with coexisting incisional hernia. Additionally, in each type one should note whether the hernia is primary or recurrent [11].

\section{Epidemiology}

It is generally believed that the overall numbers on stoma incidence quoted after the global "lleostomy Association" are significantly underestimated given that the association members maintain accounts for only approximately $17 \%$ of colostomy patients and $35 \%$ of ileostomy patients. Furthermore, currently available literature provides significantly varied estimations as to the incidence of late stoma complications such as parastomal hernia. In fact, numbers ranging from anywhere between $5 \%$ and $60 \%[4,6]$ have been suggested, while Goligher goes as far as to describe this type of hernia as the most common and "unavoidable" colostomy complication [2], with somewhat lower incidence usually attributed to ileostomy. Notably, the diagnostic application of ultrasound, computed tomography or magnetic resonance imaging (MRI) significantly increases the number of diagnosed cases $[6,7,12]$.

Based on SAGES data, in the USA alone there are between 87,000 and 135,000 stoma cases annually. Half of the same are definitively stomata, with approximately 20,000 to 35,000 patients (30-50\%) developing parastomal hernia. In the United Kingdom, the annual number of stomata constructed is approximately 20,000 and the national register lists 102,000 stoma cases, with around $50 \%$ of the patients developing stomal hernias, of which $30 \%$ require corrective surgery [13].

Based on the data collected by Szczepkowski in 2009 , between 5,000 and 6,000 stomata are constructed in Poland annually with the total estimated number of stoma patients reaching 20,000. The most commonly listed cases of stoma are as follows: - colorectal carcinoma (approximately 12 thousand cases) - Dukes B, C, D: 76.6\%,

- complicated diverticulosis - 20.3\%,

- inflammatory bowel disease - 0.9\%,

- anal and rectal injuries, ischaemic intestinal inflammation, intestinal stomata with urological or gynaecological indications $-2.0 \%$.

Based on the mode of surgery and the intestine involved, stoma surgery performed in Poland can be categorised as follows: urgent $53.1 \%$, planned $46.9 \%$, colostomy (usually Hartmann) $88.7 \%$, ileostomy $11.3 \%$.

In approximately $23 \%$ of the above cases parastomal hernia develops as a complication [14]. 
Based on data available from the literature globally, the incidence of hernia relative to the respective type of stoma can be summarised as follows: - end colostomy - 4.0-48.1\% (mean: 15.3\%), - loop colostomy - 0.0-30.8\% (mean: $4.0 \%$ ), - end ileostomy - 1.8-28.3\% (mean: 6.7\%), - loop ileostomy - 0.0-6.2\% (mean: 1.3\%).

As indicated in the above juxtaposition, different authors estimate the number of colostomies constructed at between $5 \%$ and $21 \%$, and most of the complications occur within the first 2 years after surgery, with the number of hernias increasing over time $[10,15,16]$.

On the basis of a review of publications available from internet databases (Medline, Pub-Med, Ovid, Google) and pertaining to stoma complications, it can be concluded that the vast majority of them are retrospective studies and there are no evidence level I publications given the absence of total and reliable data registration. What can be inferred from the available reports is the lack of significant differences in terms of the incidence of early and late complications when comparing the cases of ileostomy and colostomy. The slight discrepancy in the incidence of hernia in favour of ileostomy (9-22\%) compared to colostomy (18-40\%) can be treated as inconclusive at best given the absence of statistical significance. The subjectively best results of loop ileostomy are most likely due to its provisional character rather than any actual advantage. The characteristically significant discrepancy in terms of the number of parastomal hernia cases diagnosed results from three causes, namely the absence of objective assessment criteria, standard post-operative recovery screen systems, and standard stoma techniques. At the same time, it should be emphasised that stoma creation is not a procedure that could be performed by an inexperienced surgeon. In the efforts aimed at parastomal hernia prevention, it ought to be remembered that in emergencies, loop ileostomies are preferred over colostomies, whereas in definitive stomata the preference is towards end stomata with a careful pre-operative patient examination and marking out the location of the planned stoma [17].

A meta-analysis of 24 publications indicates the absence of a statistically significant difference in the incidence of parastomal hernia depending on the stoma location in relation to the rectus abdominis muscle; for instance, as reported by the authors quoted above, the percentage of stomas formed through the rectus abdominis muscle versus adjacent to the muscle is as follows: $3 \%$ vs. $22 \%$ (Sjodahl 1988 ), $37 \%$ vs. $33 \%$ (Williams 1990 ), $52 \%$ vs. $46 \%$ (Ortiz 1994).

What follows is that despite the prevalent belief of the lower risk of hernia associated with stoma constructed through the rectus muscle, only $1 / 6$ of studies included in the mentioned meta-analysis indicated a lower percentage of parastomal hernia for this location [10].

A separate clinical problem is the incidence of parastomal hernia recurrence following corrective surgery. Depending on the selected reconstructive technique, the results can be summarised as follows: - after surgery with stoma transposition - 0-76.2\% (mean: 24.3\%),

- after mesh plasty - 0-33.3\% (mean: 2.9\%),

- simple tissue plasty - 46-100\% (mean: 64.9\%).

Laparoscopic techniques are sometimes used in the course of parastomal hernia surgery. However, also in this case the recurrence percentages are similar (8-56\%) to comparable proportional results for the respective types of plasty [4, 10, 12, 17-19]. Unfortunately, the reports lack randomised studies and most of the papers pertain to relatively small patient samples [20-22]. Moreover, hernia recurrence may occur not only at the location of the newly formed stoma but, according to the quoted authors, there is also a $1 \%$ to $32 \%$ risk of recurrence at the location of the removed stoma [12].

The first use of laparoscopy in stoma surgery was in 1991 in a procedure performed by Lange while conducting loop colostomy. Since then, the number of stomata aided by laparoscopy has been on a steady increase, including the development of minimally invasive methods such as laparoscopic procedures involving only a single incision of the integuments - SILS [23, 24]. Unfortunately, a review of medical databases produced scarce reports on hernia incidence in stomata constructed using such techniques, and in the few available publications the follow-up periods were under 12 months. No randomised studies were available [25].

\section{Risk factors}

It is important to distinguish between surgical and patient variables to extract a full picture of the risk factors associated with the development of a parastomal hernia $[1,18,26,27]$. 
In terms of patient-dependent factors, the following parameters are typically listed as significant $[3,5,17,19]$ :

- age > 60 years (some authors mention 67 years as the age limit),

- obesity with body mass index $(\mathrm{BMI})>30 \mathrm{~kg} / \mathrm{m}^{2}$,

- waist circumference $>100 \mathrm{~cm}$,

- diabetes,

- smoking tobacco,

- systemic and local infection,

- hard physical labour as a relative risk factor,

- ASA classification > II,

- low tensile strength of abdominal wall,

- chronic cough and/or chronic obstructive pulmonary disease (COPD),

- steroid therapy,

- eating or immune disorders and collagen metabolism disorders,

- Crohn's disease,

- cancer,

- ischaemia.

Taking surgery related factors into consideration, the available data suggest that the incidence of parastomal hernia increases in time, reaching between $59 \%$ and $76 \%$ after 20 years $[28,29]$. Relative to the surgical method used in the creation of the stoma, it seems that hernia occurs more commonly in the following cases [10, 30]:

- after end colostomy, when compared to end ileostomy,

- after loop colostomy, when compared to loop ileostomy,

- after end ileostomy, when compared to loop ileostomy,

- after the trans-peritoneal approach route, when compared to the extra-peritoneal approach route.

The incidence of parastomal hernia as a recurrence after corrective surgery is as follows [12]:

a) radiological recurrence $(p=0.05)$ :

- with mesh - $22 \%$,

- without mesh - 45\%;

b) clinical recurrence $(p<0.001)$ :

- with mesh - $13 \%$,

- without mesh - 80\%.

It is believed that among surgical factors contributing to the increased incidence of parastomal hernia, the most important include [10, 17, 27]:

- urgency of surgery,

- stoma type,

- pre-operative marking of the stoma location,
- prior parastomal hernia surgery,

- surgeon's experience and qualifications,

- referral level of the healthcare institution,

- type of suture and mesh,

- stoma creation technique,

- size of the stoma orifice in integuments above $3 \mathrm{~cm}$ (the accepted optimum is $2 / 3$ of the intestine width).

A somewhat less evident correlation between the incidence of hernia and the use of the following surgical techniques can also be inferred from the literature; however, the literature review and meta-analysis did not confirm their prognostic significance $[4,10,18,26]$ :

- position of the stoma relative to the rectus muscle, - placing of the fixing sutures to the fascia,

- closing the lateral extra-intestinal space forming the stoma.

Based on the performed literature review (Medline, Pub-Med, Ovid, Google), it can be concluded that the assessment of surgical risk factors suffers from the lack of evidence level I publications and most of the available studies are retrospective [17]. One of the few available prospective studies reported that regardless of the underlying reason for constructing the stoma and the urgent or planned surgery mode, the observed incidence of parastomal hernia reached 33\% [31]. In turn, a multi-factor analysis of parastomal hernia risk factors indicated that the size of the stoma incision and the patient's age are in fact independent risk factors in this respect:

- each additional millimetre increases the risk of hernia by $10 \%(\mathrm{OR}=-1.1 ; p=0.005)$,

- each additional year increases the risk of hernia by $4 \%(\mathrm{OR}=-1.04 ; p=0.04)$

- sigmoidostomy is burdened with a significantly greater risk of hernia (45.9\%) when compared to ileostomy $(22 \%)(p<0.05)$.

The presence of other pathologies such as disseminated neoplastic disease, BMI above $35 \mathrm{~kg} / \mathrm{m}^{2}$, prostatic hyperplasia, diabetes, chronic constipation and ascites was not found to significantly correlate with the incidence of hernia.

It is commonly accepted that the incidence of parastomal hernia is highly correlated with the time since surgery. Based on this observation, the post-surgery survival should be considered, alongside wound infections, as the key post-operative risk factor $[1,18,26]$. 
Given the multilevel complexity of potential causes and the high incidence of parastomal hernia, one should draw attention to factors that can potentially minimise this risk. These include in particular [15, 32]: - adequate preoperative preparation of the patient,

- identification of high-risk patients relative to parastomal hernia incidence,

- identification of collagen metabolism disorders,

- weight loss programme,

- quitting smoking,

- physical exercise programme,

- cooperation with a stoma group,

- precise delineation of the stoma location before surgery, where possible going through the rectus muscle.

\section{Diagnosis and indications for surgery}

In the vast majority of cases, the only clinical symptom is a deformity of the abdominal wall around the stoma, which is the basis for the diagnosis. Some hernias can be overlooked due to the patient's obesity, difficulty in performing a physical examination due to severe pain during palpation, when there are contracted scars on the abdominal skin or coexisting hernias along the laparotomy incision line, and in cases of neurogenic abdominal muscle relaxation. In this respect, diagnostic imaging techniques such as ultrasound or CT may prove useful [12, 33].

It is believed that due to the continually unsatisfactory success rate of corrective surgery, indications for surgical treatment of parastomal hernia are limited mainly to cases with severe symptoms and complications, occurring in roughly $30 \%$ of all hernia patients. In this context, the main indications and contraindications for surgery are as follows [34, 35].

1. Absolute indications:

- incarceration,

- strangulation,

- obstruction,

- parastomal fistula,

- perforation,

- somal ischaemia.

2. Relative indications:

- history of incarceration,

- recurrent temporary symptoms of obstruction,

- difficulty in maintaining the collection device,

- inability to visually control and treat the stoma,

- problems with irrigation,
- hernia-related pain,

- erosion of the surrounding skin,

- inability to accept the stoma aesthetically,

- narrow hernia gate resulting in difficulty in its reduction,

- other concomitant complications with indication for intervention, e.g. stenosis or prolapsed.

3. Absolute contraindications for elective surgery:

- terminal malignant disease.

4. Relative contraindications for elective surgery:

- unresectable or metastatic cancer,

- serious comorbidity,

- scheduled temporary stoma closure.

\section{Corrective surgery techniques}

There are a number of methods that can be employed in corrective surgery depending on the approach route, possible transposition of the stoma, the use of mesh reinforcement, or the use of minimally invasive techniques.

Open techniques without prosthetic implantation can be divided into [31]:

- those without stoma transposition,

- those with stoma transposition.

Simple corrective surgery requiring only a small incision around the circumference of the stoma, approximately $5 \mathrm{~cm}$ from its edge and without transposition of the same was first described by Thorlakson in 1965 [36]. Unfortunately, high incidence of recurrence from $46 \%$ to even $100 \%$ of cases is associated with this technique, and consequently it cannot be recommended. In 1997, Bewes suggested closing the defect without producing tension by using the peritoneomuscular flap during laparotomy [37]. The risk of recurrence for this technique is $40 \%$, which is further exacerbated by the need for laparotomy and the associated risk of incisional hernia. Therefore, the technique would be justified in cases where the use of biomaterials is not possible or it is necessary to perform a laparotomy to correct additional problems.

Techniques with stoma transposition require laparotomy and are thus burdened with the additional risk of a new parastomal hernia forming, as well as of incisional hernias at the location of both the new incision and the previous stoma. Despite the above, however, transposition is associated with a lower risk of hernia recurrence compared to local reconstruction, although at the price of a somewhat increased risk of postoperative complications [38, 39]. 
Techniques with prosthetic reinforcement can be divided into [1]: open techniques, laparoscopic techniques, hybrid technique.

The first study on the use of reticular prosthetic implants in the surgical correction of parastomal hernia was published by Hopkins and Torento in 1982, nearly 30 years after the introduction of biomaterials in hernia treatment [40-42]. At first, due to the problems with non-absorbable materials inside the abdominal cavity and in contact with the internal organs, combined with the idea of placing the implant in the contaminated stoma environment, it was concluded that the use of mesh implants in parastomal hernia correction procedures is not advisable. However, to date, the available literature describes only a single case of colon erosion by the prosthetic material used in surgery [43]. With the development of biomaterials which show better integration and a lower inflammatory response the technique has gradually become the gold standard in correcting parastomal hernia [26].

Depending on the method and layer wherein the prosthetic is implanted, the currently employed surgical techniques can be classified as follows: superficial mesh (onlay technique), pre-peritoneal mesh (sublay technique), intraperitoneal mesh (inlay technique).

When the prosthetic is laid superficially, it is sutured to the margins of the aponeurosis and placed on top of it, without the need for laparotomy and with only a cutaneous incision around the stoma. Over the years, various variations of the technique have been described by Rosin and Bondari, Abdu, Leslie and Tekkis. In their publications, in patients observed over a period of 48 months, not a single case of hernia recurrence was reported [44-47].

The sublay technique may involve only a parastomal incision, as in the description by Martinez-Munive, or the need for laparotomy, as described by Cuilleret and Bou, as well as Kasperk, or even transposition of the stoma, as suggested by Alexandre and Bouillot. The authors reported a 36-month recurrence rate of $0 \%$ to $28 \%$ and no perioperative complications [48-51].

The intraperitoneal approach aims to avoid local direct access to the stoma, which theoretically reduces the risk of infection by treating the hernia intra-abdominally. One of the variations was described by Sugarbaker, wherein the mesh is not only sutured around the hernia orifice, but also forms a bridge over the extracted colon, giving the effect of peritonisation. No cases of recurrence were reported in a group of 7 patients over an observation period of 48 months [52].

Laparoscopic techniques rely on intraperitoneally implanted prosthetics. Since the first corrective surgery described by Porcheron in 1998, the number of relevant cases has been on a steady increase [20-22, 53-55]. In the method described by Hansson and named the "keyhole" technique, the mesh is cut radially and a hole to accommodate the stoma is cut in the middle. It is placed in such a way that it completely encircles the stoma from within the abdominal cavity. A laparoscopic variation on Sugarbaker's technique was described by Voitk, wherein the non-fenestrated prosthetic covers both the hernia defect and the stromal intestine [56].

A meta-analysis of 469 cases of corrective surgery using laparoscopic methods revealed that the incidence of recurrence in the case of the "keyhole" method was $27.9 \%$, while in the case of the modified Sugarbaker method it was only $10.2 \%$ [55]. Berger and Bientzle created the "sandwich technique", which combines the two aforementioned techniques by using two meshes, the first placed around the colon at the stoma site and the second to achieve "extraperitoneal" parietal positioning of the distal segment of the colon. The reported rate of recurrence for this method reaches 12\% [57]. Whichever laparoscopic technique is used, it is recommended that the mesh extends at least $5 \mathrm{~cm}$ beyond the edge of the defect [21].

In the novel surgical technique HyPER that is a combination of the laparoscopic and open approach described by Szczepkowski in 2015, the ostomy bowel is freed and delivered through a hole in the DynaMesh-IPST mesh, which is then placed intraperitoneally. It consists of four main steps: 1 laparoscopic stage; 2 - open stage; 3 -re-conversion to laparoscopic approach; 4 - final, open stage with neo-stoma formation. This procedure was carried out on twelve patients with colostomies; the mean follow-up time was 13.5 months and not a single case of hernia recurrence was reported [58].

\section{Surgical prophylaxis}

Given that the construction of a stoma invariably results in the artificial weakening of the abdominal wall, in recent years a number of studies have ap- 
peared which describe the potential benefits of the prophylactic use of a prosthetic mesh when constructing the stoma itself.

In the case of open surgery, the mesh is implanted preperitoneally, and in the case of laparoscopy, intraperitoneal implantation is preferred [13, 32, 57]. Meta-analyses of randomised studied performed until the end of 2016 with the aim of assessing the effectiveness of prophylactic prosthetic implantation during stoma construction revealed that the prophylactic approach can potentially significantly reduce the risk of parastomal hernia and does not entail complications related to the presence of the mesh itself $[59,60]$. However, the authors of one of the mentioned meta-analyses emphasized that before any conclusions are reached, one must account for the fact that the studies analysed varied considerably in terms of the employed methods and the type of stoma constructed, and that the studied patient populations were relatively small and observed over relatively short periods of time [60].

\section{Recapitulation}

It seems that to the best of modern medical knowledge, the most advantageous method of parastomal hernia correction is whichever the attending surgeon feels the most experienced with, while also being individually adapted to the needs of particular patients, their respective condition and coexisting complications. The general rule of thumb is that reinforcement of the abdominal wall with a prosthetic is the method of choice, as it promises good results and low incidence of complications or recurrence over long periods of time. The modern trend entails the use of light materials with large pores and low density. Nowadays, only the presence of a severe local parastomal septic process in a high-risk patient may be treated as a contraindication for mesh implantation and indication for performing a fast, local procedure. Local access is preferred in urgent cases and laparotomy in patients with other coexisting abdominal conditions. Laparoscopy should only be considered by adequately experienced surgeons. In the case of elective procedures in high-risk patients one should consider local parastomal hernia repair with prosthetic mesh, while laparotomy might be advised if parastomal hernia is associated with a concomitant incisional hernia. In such cases, laparoscopy is considered as an equally effective and safe alternative, in particular the modified Sugarbaker technique [55].

\section{Conflict of interest}

The authors declare no conflict of interest.

\section{References}

1. Tadeo-Ruiz G, Picazo-Yeste JS, Moreno-Sanz C, Herrero-Bogajo ML. Parastomal hernias: background, current status and future prospects. Cir Esp 2010; 87: 339-49.

2. Goligher JC. Surgery of the anus, rectum and colon. $5^{\text {th }}$ ed. Bailliere Tindall 1985; 703-5.

3. Martin L, Foster G. Parastomal hernia. Ann R Coll Surg Engl 1996; 78: 81-4.

4. Israelsson LA. Preventing and treating parastomal hernia. World J Surg 2005; 29: 1086-9.

5. Arumugam PJ, Bevan L, Macdonald L, et al. A prospective audit of stomas-analysis of risk factors and complications and their management. Color Dis 2003; 5: 49-52.

6. Moreno-Matias J, Serra-Aracil X, Darnell-Martin A, et al. The prevalence of parastomal hernia after formation of an end colostomy. A new clinico-radiological classification. Color Dis 2009; 11: 173-7.

7. Seo SH, Kim HJ, Oh SY, Lee JH, Suh KW. Computed tomography classification for parastomal hernia. J Korean Surg Soc 2011; 81: 111-4.

8. Gil G, Szczepkowski M. A new classification of parastomal hernias: from the experience at Bielanski Hospital in Warsaw. Pol Przegl Chir 2011; 83: 430-7.

9. Śmietański M, Bury K, Matyja A, et al. Polish guidelines for treatment of patients with parastomal hernia. Pol Przegl Chir 2013; 85: 152-80.

10. Carne PWG, Robertson GM, Frizelle FA. Parastomal hernia. Br J Surg 2003; 90: 784-93.

11. Śmietański M, Szczepkowski M, Alexandre JA, et al. European Hernia Society classification of parastomal hernias. Hernia 2014; 18: 1-6.

12. Cingi A, Solmaz A, Attaallah W, Aslan A, Aktan AO. Enterostomy closure site hernias: a clinical and ultrasonographic evaluation. Hernia 2008; 12: 401-5.

13. Jänes $A$, Cengiz $Y$, Israelsson LA. Randomized clinical trial of the use of a prosthetic mesh to prevent parastomal hernia. $\mathrm{Br}$ J Surg 2004; 91: 280-2.

14. Szczepkowski M, Borycka K, Bielecki K. Wyniki ogólnopolskiego prospektywnego badania epidemiologicznego pacjentów ze stomią. Proktologia 2009; 10: 94-107.

15. Thompson MJ. Parastomal hernia: incidence, prevention and treatment strategies. Br J Nurs 2008; 17: 18-20.

16. Rondelli F, Reboldi P, Rulli A, et al. Loop ileostomy versus loop colostomy for fecal diversion after colorectal or coloanal anastomosis: a meta-analysis. Int J Colorectal Dis 2009; 24: 479-88.

17. Shabbir J, Britton DC. Stoma complications: a literature overview. Color Dis 2010; 12: 958-64.

18. Black P. Managing physical postoperative stoma complications. Br J Nurs 2009; 18: 4-10. 
19. Nastro P, Knowles CH, McGrath A, et al. Complications of intestinal stomas. Br J Surg 2010; 97: 1885-9.

20. Hansson BME, De Hingh IHJT, Bleichrodt RP. Laparoscopic parastomal hernia repair is feasible and safe: early results of a prospective clinical study including 55 consecutive patients. Surg Endosc Other Interv Tech 2007; 21: 989-93.

21. Berger D, Bientzle M. Laparoscopic repair of parastomal hernias: a single surgeon's experience in 66 patients. Dis Colon Rectum 2007; 50: 1668-73.

22. Mancini GJ, McClusky DA, Khaitan L, et al. Laparoscopic parastomal hernia repair using a nonslit mesh technique. Surg Endosc Other Interv Tech 2007; 21: 1487-91.

23. Hasegawa J, Hirota M, Kim HM, et al. Single-incision laparoscopic stoma creation: experience with 31 consecutive cases. Asian J Endosc Surg 2013; 6: 181-5.

24. Miyoshi N, Fujino S, Ohue M, et al. Standardized technique for single-incision laparoscopic-assisted stoma creation. World J Gastrointest Endosc 2016; 8: 541-5.

25. Carne PWG, Frye JNR, Robertson GM, Frizelle FA. Parastomal hernia following minimally invasive stoma formation. ANZ J Surg 2003; 73: 843-5.

26. Israelsson LA. Parastomal hernias. Surg Clin North Am 2008; 88: 113-25

27. McGrath A, Porrett T, Heyman B. Parastomal hernia: an exploration of the risk factors and the implications. Br J Nurs 2006; 15: 317-21.

28. Cheung MT. Complications of an abdominal stoma: an analysis of 322 stomas. Aust N Z J Surg 1995; 65: 808-11.

29. Leong APK, Londono-Schimmer EE, Phillips RKS. Life-table analysis of stomal complications following ileostomy. Br J Surg 1994; 81: 727-9.

30. Rullier E, Le Toux N, Laurent C, et al. Loop ileostomy versus loop colostomy for defunctioning low anastomoses during rectal cancer surgery. World J Surg 2001; 25: 274-7.

31. Pilgrim CHC, McIntyre R, Bailey M. Prospective audit of parastomal hernia: prevalence and associated comorbidities. Dis Colon Rectum 2010; 53: 71-6.

32. Berger D. Prevention of parastomal hernias by prophylactic use of a specially designed intraperitoneal onlay mesh (Dynamesh IPST $^{\oplus}$. Hernia 2008; 12: 243-6.

33. Cingi A, Cakir T, Sever A, Aktan AO. Enterostomy site hernias: a clinical and computerized tomographic evaluation. Dis Colon Rectum 2006; 49: 1559-63.

34. Allen-Mersh TG, Thomson JPS. Surgical treatment of colostomy complications. Br J Surg 1988; 75: 416-8.

35. Morris-Stiff G, Hughes LE. The continuing challenge of parastomal hernia: failure of a novel polypropylene mesh repair. Ann R Coll Surg Engl 1998; 80: 184-7.

36. Thorlakson RH. Technique of repair of herniations associated with colonic stomas. Surg Gynecol Obstet 1965; 120: 347-50.

37. Bewes PC. Parastomal hernia. Ann R Coll Surg Engl 1997; 79 154-5.

38. Cheung MT, Chia NH, Chiu WY. Surgical treatment of parastomal hernia complicating sigmoid colostomies. Dis Colon Rectum 2001; 44: 266-70.

39. Rubin MS, Schoetz Jr DJ, Matthews JB. Parastomal hernia is stoma relocation superior to fascial eepair? Arch Surg 1994; 129: 413-9.
40. Hopkins TB, Trento A. Parastomal ileal loop hernia repair with marlex mesh. J Urol 1982; 128: 811-2.

41. Moore TC, Siderys $H$. The use of pliable plastics in the repair of abdominal wall defects. Ann Surg 1955; 142: 973-9.

42. Koontz AR, Kimberly RC. Tantalum and marlex mesh (with a note on marlex thread): an experimental and clinical comparison: preliminary report. Ann Surg 1960; 151: 796-804.

43. Aldridge AJ, Simson JNL. Erosion and perforation of colon by synthetic mesh in a recurrent paracolostomy hernia. Hernia 2001; 5: 110-2.

44. Rosin JD, Bonardi RA. Paracolostomy hernia repair with Marlex mesh: a new technique. Dis Colon Rectum 1977; 20: 299-302.

45. Abdu RA. Repair of paracolostomy hernias with Marlex mesh. Dis Colon Rectum 1982; 25: 529-31.

46. Leslie D. The parastomal hernia. Surg Clin North Am 1984; 64: 407-15.

47. Tekkis PP, Kocher HM, Payne JG. Parastomal hernia repair: modified Thorlakson technique, reinforced by polypropylene mesh. Dis Colon Rectum 1999; 42: 1505-8.

48. Martinez-Munive A, Medina-Ramirez Llaca O, Quijano-Orvananos $F$, et al. Intraparietal mesh repair for parastomal hernias. Hernia 2000; 4: 272-4.

49. Bou CJ. Treitement deséventrations yuxtastomiales par voie intraperitoneale sans transpositions. Technique el premiers résultats. Lyon Chir 1990; 86: 577-89.

50. Kasperk R, Klinge U, Schumpelick V. The repair of large parastomal hernias using a midline approach and a prosthetic mesh in the sublay position. Am J Surg 2000; 179: 186-8.

51. Alexandre JH, Bouillot JL. Paracolostomal hernia: repair with use of a Dacron prosthesis. World I Surg 1993; 17: 680-2.

52. Sugarbaker P. Peritoneal approach to prosthetic mesh repair of paraostomy hernias. Ann Surg 1985; 201: 344-6.

53. Porcheron J, Payan B, Balique JG. Mesh repair of paracolostomal hernia by laparoscopy. Surg Endosc 1998; 12: 1281.

54. Hansson BME, Van Nieuwenhoven EJ, Bleichrodt RP. Promising new technique in the repair of parastomal hernia. Surg Endosc Other Interv Tech 2003; 17: 1789-91.

55. DeAsis F, Lapin B, Gitelis ME, Ujiki MB. Current state of laparoscopic parastomal hernia repair: a meta-analysis. World J Gastroenterol 2015; 21: 8670-7.

56. Voitk A. Simple technique for laparoscopic paracolostomy hernia repair. Dis Colon Rectum 2000; 43: 1451-3.

57. Berger D, Bientzle M. Polyvinylidene fluoride: a suitable mesh material for laparoscopic incisional and parastomal hernia repair! Hernia 2009; 13: 167-72.

58. Szczepkowski M, Skoneczny P, Przywozka A, et al. New minimally invasive technique of parastomal hernia repair - methods and review. Videosurgery Miniinv 2015; 10: 1-7.

59. Zhu J, Pu Y, Yang X, et al. Prophylactic mesh application during colostomy to prevent parastomal hernia: a meta-analysis. Gastroenterol Res Pract 2016; 2016: 1694265.

60. Chapman SJ, Wood B, Drake TM, et al. Systematic review and meta-analysis of prophylactic mesh during primary stoma formation to prevent parastomal hernia. Dis Colon Rectum 2017; 60: 107-15.

Received: 22.06.2017, accepted: 17.09.2017. 